\title{
Current Recommendations on Treatment of Acute Diverticulitis - Mini Review
}

\author{
Jurij Janě̌* \\ Universitiy Medical Centre Ljubljana, Department of Abdominal Surgery, Slovenia
}

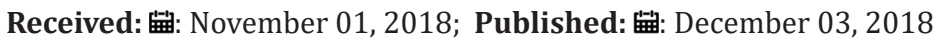

*Corresponding author: Jurij Janež, University Medical Centre Ljubljana, Department of Abdominal Surgery, Zaloška Cesta 7, 1525 Ljubljana, Slovenia

\section{Introduction}

Diverticulosis is a common disease in Western world and is associated with a Western lifestyle with low fiber diet, smoking, constipation. It is quite rare in the East, however it's incidence is growing also in the Eastern countries. Diverticula are a small outpouching of the colonic wall and are harmless, unless they became inflammed or cause other symptoms. Colonic diverticula can occur anywhere in the colon or rectum, but they most often occur in the descending and sigmoid colon [1]. The majority of individuals $(80-85 \%)$ with colonic diverticula remain asymptomatic, $10-15 \%$ of people with colonic diverticulosis develop abdominal symptoms, such as abdominal pain, bloating or changes in bowel habits. Those symptoms, without macroscopical changes within diverticula, we describe as a symptomatic uncomplicated diverticular disease (SUDD). The other complications to diverticular disease are acute inflammation of the one or more diverticula (acute diverticulitis), bleeding, fistulas with other organs, intestinal obstruction. The lifetime risk of developing acute diverticulitis in people with colonic diverticulosis is about 4\% [2]. This mini-review article summarizes some latest guidelines and suggestions regarding treatment of acute divericulitis, which were proposed mainly by the World Society of Emergency Surgeons (WSES) [3].

\section{Mini Review}

Diverticulum is the protrusion of the inner layer of the intestinal wall (mucosa and submucosa) through the weak points in the muscular layer of the intestinal wall, forming small pouches (diverticula) that bulge out the large bowel. Inflammation of diverticula is defined as diverticulitis and is most sommon in the sigmoid colon [4]. Acute diverticulitis can be simply classified as uncomplicated and complicated. In uncomplicated acute diverticulitis the inflammation is limited to the bowel wall and does not spread beyond the visceral peritoneum. Complicated diverticulitis is, when the inflammation spreads beyond the bowel wall with formation of pericolic or distant abscesses or perforation in to the abdominal cavity with consequent diffuse purulent or stercoral peritonitis [5]. The most simple and understandable classification of complicated diverticulitis was proposed by Hinchey, which classifies complicated diverticulitis in to four stages. Stage 1 is a acute diverticulitis with a pericolic abscess, stage 2 is acute diverticulitis with a distant intra-abdominal abscess, pelvic abscess or retroperitoneal abscess. Hinchey stage 3 describes complicated acute diverticulitis with diffuse purulent peritonitis and stage 4 with diffuse stercoral peritonitis. Hinchey classification helps us to decide, whether to treat patients conservatively or more invasively with percutaneous drainage or surgery.

Uncomplicated acute diverticulitis can be managed conservatively with antibiotics. Some authors believe, that mild cases of uncomplicated acute diverticulitis are self-limited and don邓t need antibiotic therapy. Mild cases of uncomplicated acute diverticulitis don邓t need admission to hospital but need to be carefully followed-up [6]. Approximately 15-20\% of patients have complicated acute diverticulitis and have an abscess on CT scan, when they are admitted to hospital. Patients with smaller diverticular abscesses (less than $4-5 \mathrm{~cm}$ ) may be treated by antibiotics alone. Patients with larger abscesses (more than 4-5 cm) can best be treated by percutaneous drainage combined with antibiotic therapy. Whenever percutaneous drainage of the abscess is not feasible or not available, based on the clinical conditions patients with large abscesses can be initially treated by antibiotic therapy alone. However, careful clinical monitoring is mandatory [3]. In Hinchey stage 3 and 4 acute diverticulitis surgical management is mandatory. Some authors recommend minimally invasive procedure with only laparoscopic lavage and drainage of the abdominal cavity in Hinchey stage 3 , when there is present purulent peritonitis without evident hole in the colon. 
However, further studies need to be done to elucidate the role of minimally invasive surgery in complicated diverticulitis. The other option, which is currently the gold standard for treatment of a perforated acute diverticulitis with diffuse peritonitis, is standard open surgery with removal of the diseased colon and formation of terminal colostomy (Hartmann®s procedure), irrigation and drainage of the abdominal cavity $[3,4,6]$. The colostomy can be temporary or permanent, depending on the patient $\ \mathrm{~s}$ age, general condition, comorbidities and also on patient邓s desire to reconstruct the large bowel. In young and healthy patients with acute perforated diverticulitis some authors recommend one stage procedure with primary anastomosis, however there is a risk of anastomotic dehiscence and another operation with formation of the terminal colostomy [3]. Even in young and healthy patients with massive peritoneal contamination it is the best option to perform two-stage procedure with Hartmann囚s procedure first and terminal colostomy closure later [3-6].

\section{Conclusion}

Acute diverticulitis is a complication of diverticulosis. It can be uncomplicated or complicated. Uncomplicated forms of acute diverticulitis can usually be managed conservatively. Complicated acute diverticulitis can also be managed concervatively with antibiotics or in combination with percutaneous drainage. Surgical management must be employed for advanced forms of complicated acute diverticulitis with perforation and peritonitis.

\section{References}

1. Cabotti M, Annibale B (2018) Treatment of diverticular disease: an update on latest evidence and clinical implications. Drugs in Context 7: 212526.

2. Oh HK, Han EC, Ha HK, Choe EK, Moon SH, et al. (2014) Surgical management of colonic diverticular disease: discrepancy between rightand left-sided diseases. World J Gastroenterol 20(29): 10115-10120.

3. Sartelli M, Catena F, Ansaloni L, Coccolini F, Griffiths EA, et al. (2016) WSES Guidelines for the management of acute left sided colonic diverticulitis in the emergency setting. World Journal of Emergency Surgery 11: 37.

4. Abraha I, Binda GA, Montedori A, Arezzo A, Cirocchi R (2017) Laparoscopic versus open resections for the sigmoid diverticulitis (Review). Cochrane Database of Systematic Reviews 11: CD009277.

5. Zizzo M, Maneti A, Ugoletti L (2018) Current treatment of acute perforated diverticulitis: the role of damage control surgery. Journal of Inflammation Research 11: 319-320.

6. Gemlo BT (2004) Elective Surgical Treatment of Diverticulitis. Clinics in Colon and Rectal Surgery 17(3):183-186.
ISSN: 2574-1241

DOI: 10.26717/BJSTR.2018.11.002132

Jurij Janež. Biomed J Sci \& Tech Res

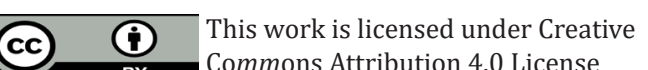

Submission Link: https://biomedres.us/submit-manuscript.php

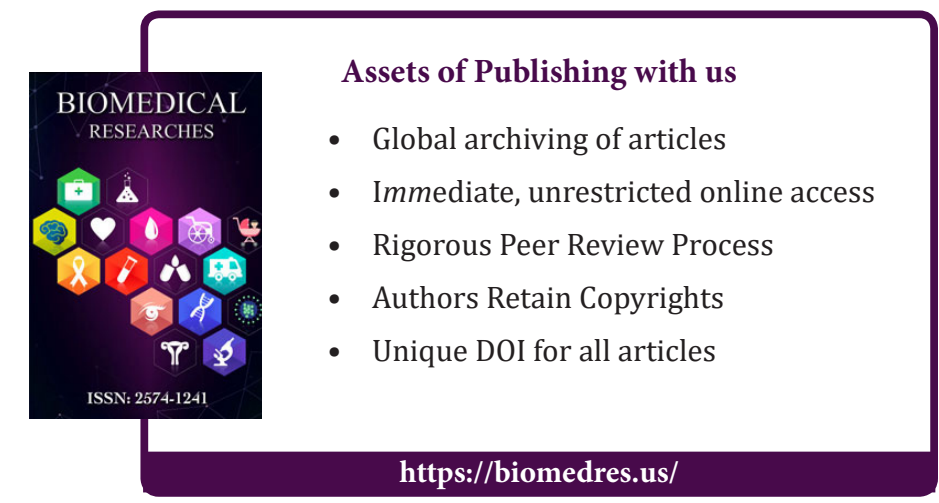

